\title{
Clinical and molecular characterization of HER2 amplified-pancreatic cancer
}

Angela Chou ${ }^{1,2,3+}$, Nicola Waddell ${ }^{6 \dagger}$, Mark J Cowley ${ }^{1,3+}$, Anthony J Gill ${ }^{1,4,5}$, David K Chang ${ }^{1,12,13}$, Ann-Marie Patch ${ }^{6}$, Katia Nones ${ }^{6}$, Jianmin Wu ${ }^{1,3}$, Mark Pinese ${ }^{1,3}$, Amber L Johns ${ }^{1}$, David K Miller ${ }^{6}$, Karin S Kassahn ${ }^{6}$, Adnan M Nagrial ${ }^{1,3}$, Harpreet Wasan ${ }^{7}$, David Goldstein ${ }^{8}$, Christopher W Toon ${ }^{4,5,9}$, Venessa Chin ${ }^{1,3}$, Lorraine Chantrill ${ }^{1,3,10}$,

Jeremy Humphris ${ }^{1}$, R Scott Mead ${ }^{1,2,3}$, Ilse Rooman ${ }^{1,3}$, Jaswinder S Samra ${ }^{11}$, Marina Pajic ${ }^{1,3}$, Elizabeth A Musgrove ${ }^{1,12}$, John V Pearson ${ }^{6}$, Adrienne L Morey ${ }^{2^{*}}$, Sean M Grimmond ${ }^{6,12^{*}}$ and Andrew V Biankin ${ }^{1,12,13^{*}}$

\begin{abstract}
Background: Pancreatic cancer is one of the most lethal and molecularly diverse malignancies. Repurposing of therapeutics that target specific molecular mechanisms in different disease types offers potential for rapid improvements in outcome. Although HER2 amplification occurs in pancreatic cancer, it is inadequately characterized to exploit the potential of anti-HER2 therapies.

Methods: HER2 amplification was detected and further analyzed using multiple genomic sequencing approaches. Standardized reference laboratory assays defined HER2 amplification in a large cohort of patients $(n=469)$ with pancreatic ductal adenocarcinoma (PDAC).

Results: An amplified inversion event (1 MB) was identified at the HER2 locus in a patient with PDAC. Using standardized laboratory assays, we established diagnostic criteria for HER2 amplification in PDAC, and observed a prevalence of 2\%. Clinically, HER2- amplified PDAC was characterized by a lack of liver metastases, and a preponderance of lung and brain metastases. Excluding breast and gastric cancer, the incidence of HER2-amplified cancers in the USA is $>22,000$ per annum.

Conclusions: HER2 amplification occurs in 2\% of PDAC, and has distinct features with implications for clinical practice. The molecular heterogeneity of PDAC implies that even an incidence of $2 \%$ represents an attractive target for anti-HER2 therapies, as options for PDAC are limited. Recruiting patients based on HER2 amplification, rather than organ of origin, could make trials of anti-HER2 therapies feasible in less common cancer types.
\end{abstract}

\section{Background}

Pancreatic cancer is the fourth leading cause of cancer death in western societies, with a 5-year survival rate of less than $5 \%$ [1]. Systemic therapies are only modestly effective; however, there is emerging evidence that small groups of patients may respond well to specific treatments $[2,3]$. Current therapeutic development is focused on targeting molecular mechanisms, and this has resulted

\footnotetext{
* Correspondence: amorey@stvincents.com.au; s.grimmond@imb.uq.edu.au; andrew.biankin@glasgow.ac.uk

${ }^{\dagger}$ Equal contributors

${ }^{2}$ Anatomical Pathology, Sydpath, St Vincent's Hospital, Sydney, Australia

${ }^{6}$ Queensland Centre for Medical Genomics, Institute for Molecular Bioscience,

University of Queensland, Brisbane, Australia

${ }^{1}$ Kinghorn Cancer Centre and Garvan Institute of Medical Research,

Darlinghurst, Sydney, Australia

Full list of author information is available at the end of the article
}

in significant improvements in outcome for several cancer types (for example, crizotinib for EML4-ALK fusion-positive non-small cell lung cancer (NSCLC)). This approach is shifting the traditional organ-based classification of cancer towards a new molecular taxonomy, and creating opportunities to apply therapeutics for the treatment of cancers originating in other organs that harbour similar molecular anomalies. Such indications for extension of existing therapeutics is attractive; however, specific molecular phenotypes and diagnostic characteristics are complex and usually inadequately defined $[4,5]$. The target population for cancers of organs, apart from where the therapeutic strategy was initially developed, often occur at low frequency, further adding to the challenge.

\section{Biomed Central}


Emerging data from cancer sequencing initiatives such as the International Cancer Genome Consortium (ICGC) [6] and The Cancer Genome Atlas (TCGA) [7] are unveiling a vast heterogeneity of molecular aberrations in cancer. Pancreatic ductal adenocarcinoma (PDAC), the predominant form of pancreatic cancer, is particularly heterogeneous, and apart from a few notable exceptions, which have not been successfully targeted, most genetic aberrations have a frequency of $2 \%$ or less [8-10].

Trastuzumab, a monoclonal antibody that targets the HER2 receptor, is an effective therapy for HER2-amplified breast cancer, and was recently extended to the treatment of HER2-amplified gastric cancer [11]. In addition, borderline signals have been seen in clinical trials of semi-selected patients with NSCLC [4], and case reports describe exceptional responses to trastuzumab in other cancers with HER2 amplification, such as cholangiocarcinoma [12]. Defining HER2 amplification as a biomarker of trastuzumab responsiveness is integral to targeting appropriate populations for therapy. Although HER2 overexpression and amplification has been assessed in PDAC (see Additional file 1), standardized diagnostic assays have on the whole not been applied, and the roles of emerging diagnostic approaches such as genomic sequencing are yet to be explored. As a consequence, the diagnostic criteria and prevalence of HER2 amplification in PDAC remain unclear. Although preclinical studies support potential efficacy of trastuzumab in PDAC [13,14], clinical trials have been hampered by non-standardized assays and a consequent lack of focus on appropriate subgroups $[15,16]$. With the current poor survival, low therapeutic responsiveness, and vast molecular heterogeneity of PDAC, even if a relatively low proportion were found to be HER2-amplified, targeting by HER2 amplification represents an attractive potential therapy.

In this study, we identified HER2 amplification in diagnostic specimens of PDAC using single nucleotide polymorphism (SNP) arrays and whole genome sequencing. We defined the characteristics and prevalence (2.1\%) of HER2 amplification in a large cohort of patients with resected PDAC using standardized reference laboratory assays. We found that HER2-amplified PDAC has an atypical pattern of metastatic spread with a predilection for lung metastasis and local recurrence, rather than liver metastases. Assessment of HER2 amplification across 16 cancer types suggested a prevalence of at least 22,000 cases per annum in the USA (excluding breast and gastric cancer), suggesting that a molecular recruitment strategy may make it feasible to test anti-HER2 therapies in less common cancer types.

\section{Methods}

\section{Ethics approval}

Ethics approval for acquisition of data and biological material was obtained from the human research ethics committee at each participating institution, conducted in accordance with the National Statement on Ethical Conduct in Human Research 2007 and the Declaration of Helsinki. Consent was obtained from prospectively recruited patients for genomic sequencing through the Australian Pancreatic Cancer Genome Initiative (APGI) as part of the ICGC. Consent was waived by Human Research Ethics Committees for retrospectively acquired data and material under an approved protocol (see Additional file 2).

\section{Genomic sequencing, copy number, and mRNA expression analysis}

Patients were prospectively recruited to the APGI [17] for genomic sequencing as part of the ICGC, and details of sample acquisition and processing have been described previously [9]. Briefly, samples focused on primary operable non-pretreated PDAC. Tissue was prepared by either full face frozen sectioning or the ends being excised and processed in formalin, then representative sections were reviewed by at least one pathologist to verify presence of carcinoma in the sample to be sequenced, and to estimate the percentage of malignant epithelial nuclei in the sample relative to stromal nuclei. Nucleic acids were extracted from fresh frozen tumour and normal tissue pairs, and whole genome and exome sequencing was performed using a combination of long mate pair and paired-end approaches. DNA copy number was assessed using specific microarrays (HumanOmni1-Quad BeadChip, Illumina Inc., San Diego, CA, USA). Primary tumour mRNA expression was assayed using human microarrays (HT-12 V4; Illumina) (GEO accession GSE36924). Gene expression profiles were classified into intrinsic breast cancer subtypes using the PAM50 classifier [18].

\section{Defining prevalence and diagnostic criteria for HER2 amplification}

Formalin-fixed, paraffin wax-embedded diagnostic material from a cohort of 469 patients who had undergone operative resection for PDAC was accrued from 12 institutions associated with the APGI between 1990 and 2012. Subsets of this cohort have previously been published $[19,20]$, and detailed characteristics are presented (see Additional file 3: Table S2).

HER2 immunohistochemistry (IHC) and in situ hybridization (ISH) was performed in a national reference HER2 diagnostic testing laboratory. Scoring for HER2 protein expression and amplification was based on criteria recommended for breast and gastric carcinoma (Table 1) [21].

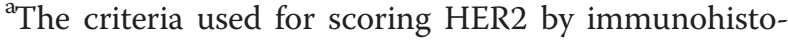
chemistry and in situ hybridization in PDAC were as follows. Her2 IHC score criteria (modified from Hofmann et al [21]): 0, no staining of any pattern or intensity; $1+$. weak discernable membrane staining; $2+$, mild to 
Table 1 IHC and FISH scoring for the detection of HER2 amplification $^{\mathrm{a}}$

\begin{tabular}{llcc}
\hline Her2 IHC & IHC scores & \multicolumn{2}{c}{ HER2 FISH } \\
\cline { 3 - 4 } score & ( $\mathbf{n}=\mathbf{4 6 9 )}$ & Positive, $\mathbf{n = 1 0}$ & Negative, $\mathbf{n = 4 5 9}$ \\
\hline $3+$ & $7(1.5 \%)$ & 7 & 0 \\
$2+$ & $27(5.8 \%)$ & 3 & 24 \\
$1+$ & $59(12.6 \%)$ & 0 & 59 \\
0 & $376(80.2 \%)$ & 0 & 376 \\
\hline
\end{tabular}

Abbreviations: FISH fluorescence in situ hybridization; IHC

immunohistochemistry; PDAC pancreatic ductal adenocarcinoma.

moderate complete or basolateral membrane staining; $3+$, strong, complete, or basolateral membrane staining. HER2 ISH criteria: non-amplified, Her2 count $<4$ and Her2: cep17 ratio <2; amplified, Her2 count $\geq 4$ and Her2:cep17 ratio $\geq 2$.

\section{Results}

In the initial set of 50 patients recruited to the APGI, SNP arrays detected a case of HER2 amplification (Figure 1A), with associated high HER2 mRNA expression (see Additional file 4). HER2 amplification was verified using standardized laboratory assays currently used for breast and gastric cancer at an HER2 diagnostic reference laboratory. This case was analyzed further using whole genome sequencing, copy number analysis, and mRNA expression profiling.

\section{Genomic characteristics of HER2-amplified pancreatic ductal adenocarcinoma}

Whole genome and exome sequencing identified several classes of mutation including single nucleotide variants, small insertions and deletions (indels), and chromosomal rearrangements (see Additional file 5). Chromosomal rearrangements included a region of loss on chromosome 18 containing the MAPK4,DCC, SMAD4, and $E L A C 1$ genes, and three complex amplification regions: an inverted amplification of a region of chromosome 17, spanning HER2 and truncating MED1 and TOP2A at either end (chr17:37,565,271-38,554,848) (Figure 1B), and two overlapping fold back inversion events on chromosome 9: a $1.5 \mathrm{Mb}$ event within a $1.9 \mathrm{Mb}$ event, disrupting $U B A P 2$. Exome sequencing identified 23 somatic mutations affecting 21 genes, including $K R A S^{\text {p.G12V }}$ and $T P 53^{\mathrm{p} \cdot \mathrm{Q} 317}$, and 2 mutations in both TOP2A and RYR2 (Figure 1C).

We performed gene set enrichment analysis using all genes affected by mutation, amplification, copy number alterations (GISTIC2.0 peaks $P<0.25$ ), and disruptions caused by structural rearrangements (see Additional file 6). There were three strong biological themes: 1 ) genes from the HER2-amplicon (13 genes; $\left.P<1 \times 10^{-7}\right) ; 2$ ) a broad cancer theme driven by KRAS, TP53, and EPHA5 (13 gene sets; $P<0.001)$; and 3 ) an immune signature, driven by type I interferons, IFNA2, INFA6, INFA13 (7 gene sets; $P<0.001$ ), which are involved in recognition of viral infection and neoplasia (see Additional file 7).

Intrinsic subtype analysis using expression microarray data from the APGI cohort $(\mathrm{n}=90)$ [9] classified each PDAC sample using the PAM50 classifier, which captures five breast cancer subtypes: HER2-amplified, luminal A, luminal B, basal, and normal-like [18]. The HER2amplified patient with PDAC identified above clustered with the HER2-amplified intrinsic subtype, with a confidence of $93 \%$. This was driven by high expression of HER2, GRB7, and FOXA1, and low expression of KRT17 and MIA (see Additional file 8).

\section{HER2 amplification in pancreatic ductal adenocarcinoma}

In order to define the clinical and histopathological characteristics, diagnostic criteria, and prevalence of HER2 amplification in PDAC, we assessed a cohort of 469 patients with resected PDAC using IHC and in situ hybridization (ISH). IHC for Her2 protein expression showed $3+$ staining in 7 cases (1.5\%) (Figure 2A), 2+ staining in $27(5.8 \%), 1+$ in $59(12.6 \%)$ and no staining in 376 (80.2\%) (Table 1). All 7 cases that exhibited Her2 3+ staining were found to be HER2-amplified on silver ISH and fluorescence ISH (FISH) with 100\% concordance between the two methods (Figure 2B,C). Only 3 of 27 cases that were Her2 2+ on IHC were found to be HER2amplified (11\%). No cases of $1+$ or negative Her2 IHC were HER2-amplified, and the overall prevalence of HER2 amplification was $2.1 \%$ (10 of 469 cases). All the amplified cases showed a tendency for the HER2 signal to form clusters. One case was found to have intra-tumoral heterogeneity by IHC and ISH, with distinct HER2-positive and HER2-negative areas. As HER2 amplification was only present when Her2 IHC produced $2+$ or $3+$ staining, IHC screening followed by ISH in cases of $2+$ and $3+$ IHC staining may be an appropriate and cost-effective approach for the detection of HER2-amplified PDAC (see Additional file 9).

\section{Clinical features of HER2-amplified pancreatic ductal adenocarcinoma}

Of the ten patients with HER2 amplified tumours, nine died of PDAC, and one died of respiratory failure as a result of chronic obstructive pulmonary disease with no evidence of cancer recurrence. The pattern of metastatic relapse of the HER2-amplified cases was characterized by a lack of liver metastases. None of the eight cases with a documented site of recurrence had liver metastases (one patient had recurrence, and for another patient the site of recurrence was not reported). Of these eight patients, four had lung metastases (one of whom also had brain metastases (Figure 2D-F), and two also had local 

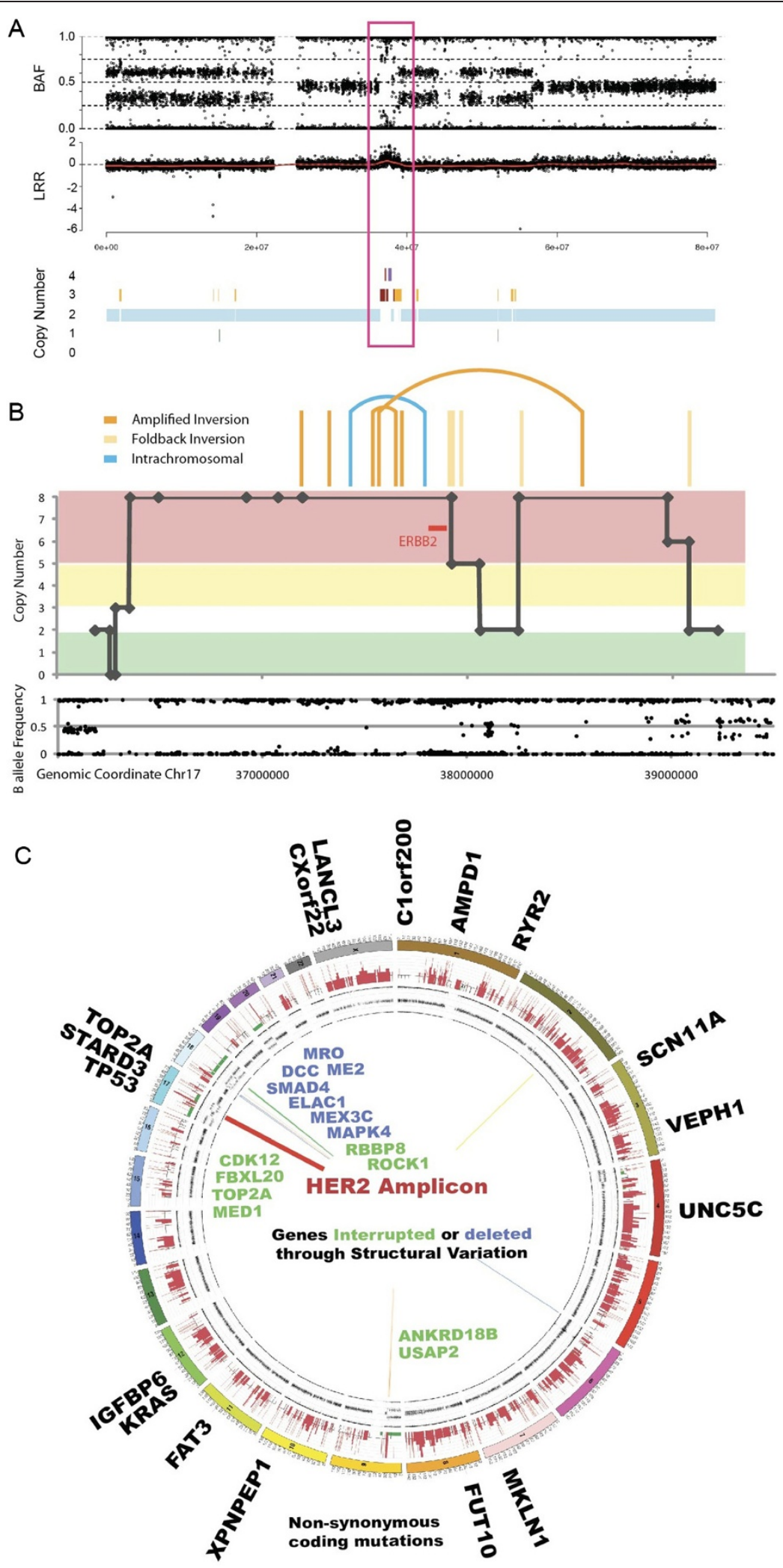

Figure 1 (See legend on next page.) 
(See figure on previous page.)

Figure 1 HER2-amplified pancreatic ductal adenocarcinoma. (A) Single nucleotide polymorphism (SNP) array showing amplification on chromosome 17 containing the HER2 locus (boxed). (B) Inverted amplification on chromosome 17, spanning HER2 and truncating MED1 and TOP2A at either end (chr17:37,565,271-38,554,848). (C) Circos plot showing mutations and structural rearrangements of patient 9 (Table 2). Genes with non-silent substitutions are shown on the outer wheel. The SNP array data are shown on the subsequent plots (copy number predictions and B-allele frequency of probes). Structural rearrangements are indicated with lines inside the circle: deletions (green), inversions (yellow), intrachromosomal rearrangements (blue), foldback inversions (light orange), and amplified inversions (dark orange). Genes with structural variation are shown in blue if deleted and green if interrupted.

recurrence, although cerebral imaging was only performed in one patient), while the remaining four patients had peritoneal and local recurrences only, although imaging of the thorax was not performed in two of these patients (Table 2). The higher prevalence of lung metastasis (in the absence of liver metastases) in HER2-amplified tumours was significantly different to that of non-HER2-amplified tumours $(P=0.0022$; Table 3$)$, as was the rate of recurrence at any site without liver metastases $(P=0.0028$; Table 3). HER2 amplification was not associated with any other clinicopathological characteristics such as age $(P=0.5467)$, sex $(P=0.7520)$, tumour stage $(P=0.4495)$, grade $(P=0.1259)$, or size $(P=0.4695)$, or disease-specific survival $(P=0.2502$; Table 3$)$. Histopathologically, there were no specific features to distinguish HER2-amplified tumours from non-HER2-amplified tumours. All HER2amplified cases were moderately differentiated, and most had a diffuse or at least focally macroglandular architecture.

Since the completion of this study, we have begun prospectively screening patients with PDAC for HER2 amplification using this diagnostic approach, and identified a further two individuals (of eleven) with HER2 amplification. The first was a primary resected cancer with no evidence of recurrent disease to date at 6 months. Subsequently, whole genome sequence analysis on this patient's tumour confirmed mutation in KRAS ${ }^{p \cdot G 12 V}$. The second patient had an atypical pattern of metastatic disease at diagnosis, with a small primary pancreatic tumour on imaging, histologically confirmed peritoneal disease in

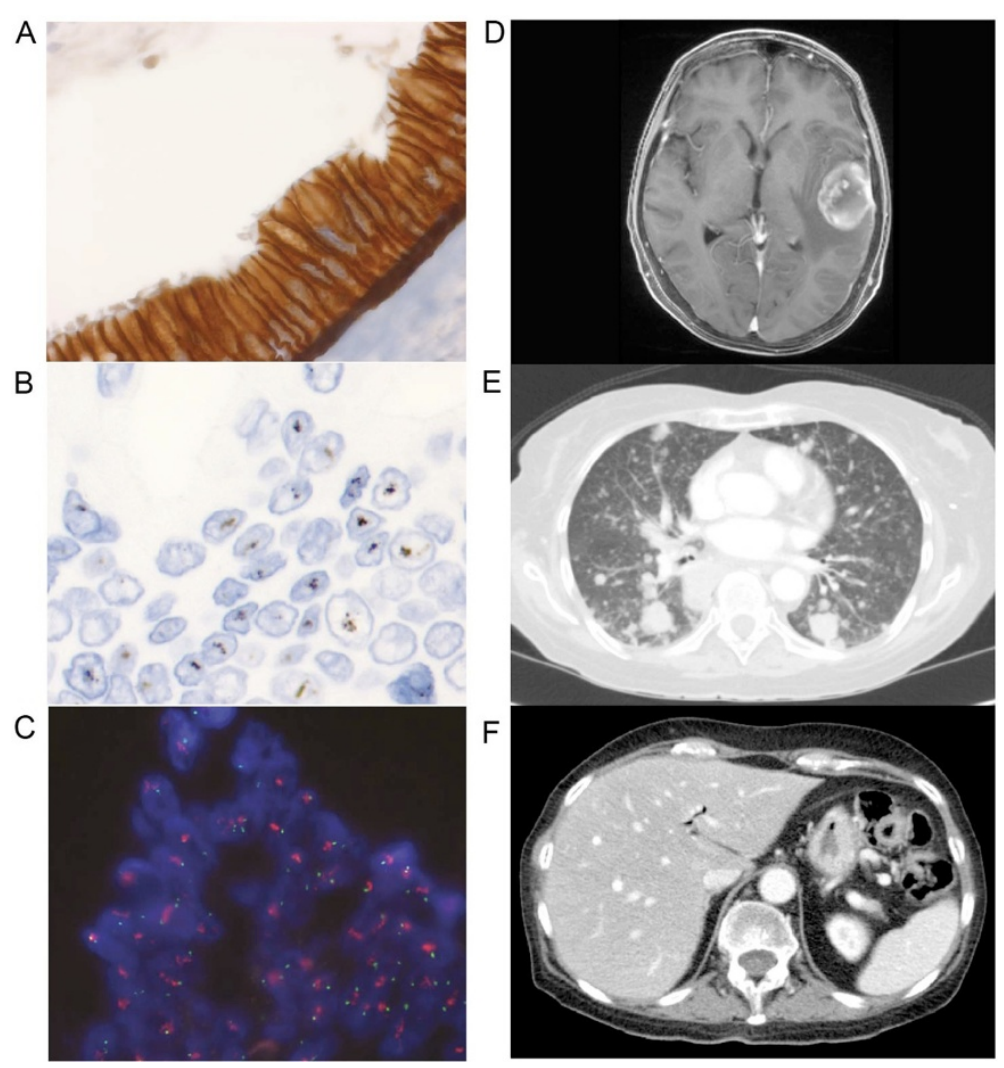

Figure 2 Clinicopathological features of HER2-amplified pancreatic ductal adenocarcinoma (PDAC). (A) Her2 immunohistochemistry showing 3+ staining, and corresponding HER2 amplification on (B) silver and (C) fluorescence in situ hybridization. Imaging results of patient 9 (Table 2) showing (D) cerebral and (E) lung metastases, and (F) absence of liver metastases. 
Table 2 Clinicopathological characteristics of HER2-amplified PDAC

\begin{tabular}{|c|c|c|c|c|c|c|c|c|c|c|c|}
\hline \multirow[t]{2}{*}{ Patient } & \multirow{2}{*}{$\begin{array}{l}\text { Her2 IHC } \\
\text { score }\end{array}$} & \multirow{2}{*}{$\begin{array}{l}\text { HER2 } \\
\text { ratio }\end{array}$} & \multirow{2}{*}{$\begin{array}{l}\text { HER2 } \\
\text { count }\end{array}$} & \multirow{2}{*}{$\begin{array}{l}\text { Overall survival } \\
\text { (months) }\end{array}$} & \multirow{2}{*}{$\begin{array}{l}\text { Cause of } \\
\text { death }\end{array}$} & \multicolumn{5}{|c|}{ Pattern of recurrence } & \multirow[t]{2}{*}{ Comments } \\
\hline & & & & & & Lung & Brain & Liver & Local & Peritoneal & \\
\hline $1^{a}$ & $3+$ & 2.45 & 6 & 13.6 & PDAC & - & ND & - & + & + & $\begin{array}{l}\text { Laparotomy showed peritoneal and local } \\
\text { recurrence }\end{array}$ \\
\hline 2 & $3+$ & 7.5 & 13 & 43.5 & COPD & - & ND & - & - & - & CT at 43 months showed no recurrence \\
\hline 3 & $3+$ & 4.12 & 10 & 5.0 & PDAC & - & ND & - & + & - & Local recurrence \\
\hline $4^{b}$ & $2+$ & 2.5 & 5.4 & 36.5 & PDAC & + & ND & - & + & + & $\begin{array}{l}\text { Ascites/pleural effusions, no liver } \\
\text { metastases on CT }\end{array}$ \\
\hline 5 & $3+$ & 4.38 & 8.1 & 18.6 & PDAC & ND & ND & ND & ND & ND & Site not documented \\
\hline 6 & 2 & 3.56 & 7 & 10.1 & PDAC & ND & ND & - & + & ND & R2 resection \\
\hline 7 & $3+$ & 2.7 & 4.5 & 41.6 & PDAC & ND & ND & - & + & + & $\begin{array}{l}\text { No liver metastases on } \mathrm{CT} \text {; peritoneal } \\
\text { recurrence }\end{array}$ \\
\hline 8 & $2+$ & 5.32 & 9 & 10.9 & PDAC & + & ND & - & + & - & No liver metastases on $\mathrm{CT}$ \\
\hline $9^{c, d}$ & $3+$ & 2.95 & 6.2 & 35.0 & PDAC & + & + & - & - & - & No liver metastases on $\mathrm{CT}$ \\
\hline $10^{e}$ & $3+$ & $2.86^{*}$ & 6.3 & 27.0 & PDAC & + & ND & - & - & - & No liver metastases on $\mathrm{CT}$ \\
\hline
\end{tabular}

Abbreviations: + present; - absent; COPD chronic obstructive pulmonary disease; CT computed tomography; FISH fluorescence in situ hybridization; IHC immunohistochemistry; ND not determined; PDAC pancreatic ductal adenocarcinoma.

aReceived adjuvant 5 -fluouracil.

${ }^{\mathrm{b}}$ Received adjuvant gemcitabine.

'Only case 9 received palliative chemotherapy (gemcitabine + erlotinib) and also received adjuvant gemcitabine.

${ }^{\mathrm{d} C a s e}$ that was whole genome sequenced.

${ }^{\text {e}}$ Case 10 showed heterogeneous staining.

the pelvis, and nodules in the lungs. Because of the unusual pattern of disease, PDAC was verified using a targeted exome panel of tissue from this patient's peritoneal disease, which showed mutations in KRAS ${ }^{\text {p.G12V }}$ and SMAD4, further supporting the diagnosis of PDAC.

\section{HER2 amplification in other cancer types}

The low prevalence of HER2 amplification in PDAC and many other cancer types makes clinical trials challenging. Analysis of genomic sequence data for 16 other cancer types (by TCGA, ICGC and other large-scale genomic efforts) identified an incidence of $0.5 \%$ to $13 \%$, which equates to over 54,000 new cases of HER2-amplified cancers per year in the USA alone (see Additional file 10). Excluding breast and gastric cancer, we estimate an incidence in excess of 22,000 HER2 amplified cancers per annum. Recruiting patients based on HER2 amplification rather than organ of tumour origin would make trials of anti-HER2 therapies feasible in less common cancer types. In order to identify these 22,000 patients, 740,000 patients would need to be screened using IHC, and of these, all $2+$ and $3+$ IHC cases $(\sim 8 \%)$ would require validation using ISH. This approach may circumvent the challenges of previous clinical trials based on organ of origin recruitment, in which there have been mixed results but some efficacy in individuals (see Additional file 11). An orthogonal approach by which a primary molecular classification is used and patients are sub-classified based on organ of origin may be more tractable.

\section{Discussion}

In this study, we found that HER2-amplified PDAC has a prevalence of $2 \%$, is detectable using contemporary genomic approaches, is associated with a clinical phenotype characterized by metastatic spread predominantly to the lungs and peritoneum with local recurrence, and can metastasize to the brain but tends to avoid the liver. It bears molecular similarities to HER2-amplified breast cancer, and is yet to be adequately assessed for potential responsiveness to anti-HER2 therapy. Multiple studies in large cohorts have shown that HER2-amplified breast cancers more commonly metastasize to the lung and the brain [22]. In PDAC, clinical trial data indicate that the first site of distant metastases occurred most commonly in the liver (50\%), and occurred in the lung in $9 \%$ of the cases [23]. Autopsy studies have reported that $80 \%$ of distant metastases are to the liver, which occurred either alone or in combination with peritoneal and or lung metastases, while metastases sparing the liver made up the rest, and occurred in the peritoneum, lung, adrenal glands, and lymph nodes [24]. Cerebral metastases were not found in these studies. In our cohort of 469 patients, the incidence of lung metastases without liver metastases was $8 \%$, comparable to previous studies. We detected only one case with brain metastases $(0.2 \%)$, although only this single patient was investigated specifically for brain metastases. If we include all 10 HER2-amplified cases with documented metastatic disease, none had evidence of liver metastases $(P=0.0028)$, and the rate of lung metastases was 50\% $(P=0.0022)$. These data suggest that HER2-amplified PDAC may have a distinct 
Table 3 Clinicopathological characteristics of HER2 amplified and HER2 non-amplified cases

\begin{tabular}{|c|c|c|c|c|}
\hline \multirow[t]{2}{*}{ Clinicopathological characteristics } & \multirow{2}{*}{$\begin{array}{l}\text { Total cohort, } \\
n=469\end{array}$} & \multicolumn{2}{|c|}{ HER2 amplification } & \multirow[t]{2}{*}{$P$-value } \\
\hline & & Amplified, $n=10$ & Non-amplified, $n=459$ & \\
\hline \multicolumn{5}{|l|}{ Sex, n (\%) } \\
\hline Male & $241(51)$ & $6(60)$ & $220(51)$ & \multirow[t]{2}{*}{$0.7520^{b}$} \\
\hline Female & $228(49)$ & $4(30)$ & $210(49)$ & \\
\hline \multicolumn{5}{|l|}{ Age, years } \\
\hline Mean & 66 & 64 & 66 & \multirow[t]{3}{*}{$0.5467^{c}$} \\
\hline Median & 68 & 69.5 & 67.5 & \\
\hline Range & 28 to 88 & 47 to 73 & 28 to 87 & \\
\hline \multicolumn{5}{|l|}{ AJCC stage, n (\%) } \\
\hline $1 a$ & $17(3.6)$ & $1(10)$ & $16(3.5)$ & \multirow[t]{5}{*}{$0.4495^{\circ}$} \\
\hline $1 b$ & $25(5.3)$ & $1(10)$ & $24(5.2)$ & \\
\hline $2 a$ & $132(28.1)$ & $3(30)$ & $129(28)$ & \\
\hline $2 b$ & $279(59.5)$ & $4(40)$ & $275(60)$ & \\
\hline 4 & $16(3.4)$ & $1(10)$ & $15(3)$ & \\
\hline \multicolumn{5}{|l|}{ T stage, $\mathrm{n}$} \\
\hline $\mathrm{T} 1$ & 32 & 1 & 31 & \\
\hline $\mathrm{T} 2$ & 64 & 2 & 62 & \\
\hline T3 & 372 & 7 & 365 & \\
\hline T4 & 1 & 0 & 1 & \\
\hline \multicolumn{5}{|l|}{ N stage, $\mathrm{n}$} \\
\hline No & 180 & 6 & 174 & \\
\hline N1 & 289 & 4 & 185 & \\
\hline \multicolumn{5}{|l|}{ AJCC grade, $n$} \\
\hline 1 (well differentiated) & 36 & 0 & 36 & \multirow[t]{4}{*}{$0.1259^{d}$} \\
\hline 2 (moderately differentiated) & 305 & 10 & 295 & \\
\hline 3 (poorly differentiated) & 125 & 0 & 125 & \\
\hline 4 (undifferentiated) & 3 & 0 & 3 & \\
\hline \multicolumn{5}{|l|}{ Tumour size, mm } \\
\hline$<=20$ & 105 & 3 & 102 & \multirow[t]{2}{*}{$0.4695^{b}$} \\
\hline$>20$ & 364 & 7 & 357 & \\
\hline \multicolumn{5}{|l|}{ Vascular invasion, n } \\
\hline Present & 222 & 2 & 220 & \multirow[t]{2}{*}{$0.1106^{b}$} \\
\hline Absent & 247 & 8 & 239 & \\
\hline \multicolumn{5}{|l|}{ Perineural invasion, $\mathrm{n}$} \\
\hline Present & 339 & 8 & 331 & \multirow[t]{2}{*}{$0.7336^{b}$} \\
\hline Absent & 130 & 2 & 128 & \\
\hline \multicolumn{5}{|l|}{ Tumour location, n } \\
\hline Head & 381 & 9 & 372 & \multirow[t]{2}{*}{$0.6961^{b}$} \\
\hline Others & 88 & 1 & 87 & \\
\hline \multicolumn{5}{|l|}{ Margins } \\
\hline Clear & 301 & 7 & 294 & \multirow[t]{2}{*}{$1.0000^{b}$} \\
\hline Involved & 168 & 3 & 165 & \\
\hline
\end{tabular}




\section{Table 3 Clinicopathological characteristics of HER2 amplified and HER2 non-amplified cases (Continued)}

\begin{tabular}{|c|c|c|c|c|}
\hline \multicolumn{5}{|l|}{ Outcome } \\
\hline Follow-up, months & 0.03 to 240 & 5.0 to 43.6 & 0.03 to 240 & $1.0000^{\mathrm{b}}$ \\
\hline Median follow-up, months & 16 & 23 & 16 & \\
\hline Death: PDAC & 369 & 8 & 361 & \\
\hline Death: other & 32 & 2 & 30 & \\
\hline Death: unknown & 16 & 0 & 16 & \\
\hline Alive & 49 & 0 & 49 & \\
\hline Lost to follow-up & 3 & 0 & 3 & \\
\hline \multicolumn{5}{|l|}{ Cancer-specific survival, mean \pm SD } \\
\hline Length, months & $20 \pm 19.43$ & $28 \pm 19.65$ & $20 \pm 19.55$ & $0.2502^{c}$ \\
\hline \multicolumn{5}{|l|}{ Recurrence } \\
\hline Present & 261 & 8 & 253 & \\
\hline Absent & 78 & 1 & 77 & \\
\hline Unknown & 130 & 1 & 129 & \\
\hline \multicolumn{5}{|l|}{ Pattern of recurrence, $n$ (\% of total n) } \\
\hline Lung without liver metastasis & $22(8.4 \%$ of 261$)$ & $4(50 \%$ of 8$)$ & $18(7.1 \%$ of 253$)$ & $0.0022^{b}$ \\
\hline Any recurrence without liver metastasis & $127(49 \%$ of 261$)$ & $8(100 \%$ of 8$)$ & $119(47 \%$ of 253$)$ & $0.0028^{b}$ \\
\hline \multicolumn{5}{|l|}{ Adjuvant therapy, n } \\
\hline Yes & 175 & 3 & 172 & \\
\hline No & 289 & 7 & 282 & \\
\hline Unknown & 5 & 0 & 5 & \\
\hline
\end{tabular}

AJCC, American Joint Committee on Cancer; PDAC, pancreatic ductal adenocarcinoma.

${ }^{a}$ AJCC Cancer Staging Manual, seventh edition.

${ }^{b}$ Fisher's exact test.

'Unpaired $t$-test.

${ }^{d} X^{2}$ test.

clinical phenotype, and that liver metastases are not determined by physical factors such as portal blood flow, but by the pathophysiology of disease.

These findings have significant clinical implications. First, the detection of small lung nodules should not delay the diagnosis of metastatic disease originating in the pancreas or at relapse if the liver and other sites are clear, particularly in known HER2-amplified cases. Second, if an individual is known to have an HER2-amplified PDAC, then monitoring for disease progression in nontraditional sites such as the lung and the brain, with vigilance for neurological symptoms may be prudent. Finally, there is potential for anti-HER2 therapies in this subset of patients.

In situ hybridization studies in our reference laboratory identified HER2 amplification only in PDACs with high protein expression by IHC (score $2+/ 3+$ ). Therefore, a reasonable and cost-effective approach to universal HER2 screening is to initially test all cases with IHC and then perform secondary ISH testing only on cases with $2+/ 3+$ staining, as was initially performed for breast cancer. Using this approach, $8 \%$ of PDAC (2+ and $3+$ cancers) will require HER2 ISH assessment, and of these one-quarter will be amplified.

In the current study, in-depth genomic analysis, apart from HER2 amplification, did not reveal any features that are atypical of PDAC, with mutations of KRAS and TP53 and loss of SMAD4 found, although the inherent heterogeneity of PDAC makes it difficult to draw conclusions about the other mutations detected. mRNA expression profiles clustered with HER2-amplified breast cancer, suggesting that HER2 may be an important driver of carcinogenesis in this subgroup of PDAC.

It is interesting to note that all three HER2-amplified cases with available genomic data harboured the KRASp. G12V mutation. This mutation is less common than the p.G12D mutation, and accounts for $32 \%$ of KRAS mutations in PDAC versus $40 \%$ for p.G12D [9]. Given the small numbers of HER2-amplified cases, further studies of larger cohorts will be required before it can be determined if this association is sufficiently robust to be used diagnostically or targeted therapeutically.

Two clinical trials have assessed targeted trastuzumab therapy in PDAC $[15,16]$. Both are single arm phase II 
trials used in combination with gemcitabine [15] and capecitabine [16]. Although the latter performed HER2 FISH for $2+$ expressing cases, the former did not, and neither verified the $3+$ IHC cases by FISH. In addition, these were not standardized assays performed in reference laboratories, and resulted in a HER2 positive rate of over $10 \%$. This likely overestimation underpowered the trials by over $80 \%$, making a negative result uninterpretable.

\section{Conclusion}

HER2 amplification occurs in $2.1 \%$ of PDAC cases, and is associated with an atypical pattern of metastatic disease. A number of cancers of different organs including NSCLC, ovarian cancer, cholangiocarcinoma, and PDAC have well-defined low-prevalence HER2 amplification. Testing anti-HER2 therapies may not be feasible in organ groups because of this low prevalence and the likely heterogeneous response rates. However, these studies could be approached using novel adaptive clinical trials testing personalized therapeutic strategies (for example,: BATTLE [25], I-SPY [26], FOCUS 4 [27] and IMPaCT [3]), or using a molecular taxonomy or 'biotype' that recruits HER2amplified cancers irrespective of the organ in which the tumour arises (often referred to as 'basket' trials) [28], with specific attention to diagnostic criteria for patient recruitment, particularly as more effective anti-HER2 therapies emerge.

\section{Additional files}

Additional file 1: Table S1. Literature reports of Her2 positive rate in pancreatic ductal adenocarcinoma by immunohistochemistry and in situ hybridization techniques.

Additional file 2: Supplementary methods.

Additional file 3: Table S2. Clinico-pathological characteristics of the cohort.

Additional file 4: Figure S1. HER2 mRNA expression relative to cohort. Additional file 5: Table S3. Single nucleotide variations (SNVs) and small insertions or deletions (indels) within a patient with HER2 amplification, identified by whole exome sequencing, in MAF format. Table S4. Single nucleotide variations (SNVs) and small insertions or deletions (indels) annotated and compared with data from dbSNP, COSMIC, TCGA Breast, Biankin et al. [9] and Jones et al. [10]. Nonsynonymous mutations were assessed for functional effects by two algorithms, Polyphen 2 and SIFT; scores close to 1.0 and 0.0 , respectively, are predicted to be probably damaging. These data have already been imported into COSMIC, thus many of the COSMIC identification numbers are unique only to this patient. Table S5: Genes affected by structural variation, as determined by whole genome sequencing of tissue from a patient with HER2 amplification. Table S6: Structural and copy number aberrations in a patient identified with HER2 amplification. Listed here are the genes affected by structural variation (SV) as determined by whole genome sequencing, or copy number altered, as determined by single nucleotide polymorphism (SNP) array analysis. Genes of interest were defined as those genes affected by SV that were expected to have a functional effect (that is, genes that were truncated, amplified (but not duplicated) or deleted); genes whose copy number was found by SNP array analysis to be greatly altered, that is, reduced to 0 , or the algorithm's maximum of four copies (see Methods); and genes that were affected by copy number variation and that overlapped the statistically significant copy number altered regions, as determined by GISTIC 2.0 analysis (see Biankin et al., [9]), reported here by their q-value.

Additional file 6: Table S7. Genomic alterations within a patient identified with HER2 amplification. Genes of interest were defined as those affected by non-synonymous single nucleotide variation (SNV); small insertions or deletions (see Additional file 5: Table S3); genes affected by structural variation (SV) as determined by whole genome sequencing, or copy number variation (CNV) as determined by SNP array analysis (see Additional file 5: Table S6).

Additional file 7: Table S8. MSigDB analysis of all genes affected by mutation, tructural variation (SV) and copy number variation (CNV) (from Additional file 6: Table 57).

Additional file 8: Figure S2. Gene expression profiles from 90 pancreatic cancer primary tumors, classified into five intrinsic breast subtypes by the PAM50 classifier. The HER2-amplified patient is highlighted by a white box; genes overexpressed or underexpressed in this patient are highlighted in red and green, respectively.

Additional file 9: Figure S3. Algorithm for diagnostic testing for HER2 amplification.

Additional file 10: Table S9. Incidence of and deaths from HER2amplified cancers in the USA. Extrapolating from the frequency of HER2 amplifications identified from current projects from The Cancer Genome Atlas (TCGA) and our own International Cancer Genome Consortium (ICGC) pancreatic ductal adenocarcinoma (PDAC) project, with the estimated number of new cancer cases in the USA in 2012. From the 1.37 million expected cases, we would expect 54,000 (3.94\%) new cases of HER2-amplified cancer. Excluding breast and stomach, for which HER2 diagnostic assays are common practice, we expect 740,000 new cases, 22,000 of which will be HER2-amplified. Extrapolating from the number of immunohistochemistry (IHC) and in situ hybridization (ISH) assays to identify HER2-amplified PDAC (see Additional file 9: Figure S3), to identify these additional cases, we would expect to perform $742,000 \mathrm{IHC}$ and $117,000 \mathrm{FISH}$ assays, of which 22,000 will be confirmed (that is, will have $\mathrm{IHC}$ staining of $2+$ or $3+$ and be ISH-positive). Figure S4. Prevalence of HER2 amplification ( $\geq 4$ copies) in cancer: data from The Cancer Genome Atlas (TCGA), via the cBio portal, and the International Cancer Genome Consortium (ICGC) [9].

Additional file 11: Table S10. Clinical trials of HER2-amplified cancers, excluding breast and stomach (gastric) cancers.

\section{Abbreviations}

APGI: Australian Pancreatic Genome Initiative; FISH: Fluorescence in situ hybridization; ICGC: International Cancer Genome Consortium; IHC: Immunohistochemistry; ISH: In situ hybridization; NSCLC: Non-small cell lung cancer; PDAC: Pancreatic ductal adenocarcinoma; SNP: Single nucleotide polymorphism; TCGA: The Cancer Genome Atlas.

\section{Competing interests}

JW has received honoraria received from Roche; RS has received clinical research funding received from Therapeutic Innovation Australia and Cancer Council New South Wales; DG has an uncompensated consultant/advisory role with Celgene, Bayer, and Pfizer, and has received clinical research funding from Celgene, Pfizer, and Amgen; AM is on the Roche HER2 advisory board in Australia, and had received honoraria from Roche. The remaining authors declare that they have no competing interests.

\section{Authors' contributions}

AC, NW, MJC, AJG, DKC, JW, MP, ALJ, AMN, CT, VC, LC, JH, JSS, ALM, SMG, and $A V B$ were responsible for the concept and design of the study. All authors participated in collection and assembly of data, and AC, NW, MJC, AJG, DKC, JW, KK, MP, DG, ALM, SMG and AVB were involved in data analysis and interpretation. $A J, A P G I, L C, H W, J S, A G, D K C$ and $A V B$ were involved in provision of study materials or patients: All authors were involved in manuscript writing and final approval of the manuscript. 


\section{Acknowledgments}

We thank all the members of the Australian APGI (www.pancreaticcancer.net. au/apgi/collaborators) for their continuing support with provision of biospecimens and clinical data. We also thank Mary-Anne Brancato BSc, Michelle Thomas BSC, Sarah Rowe BSc, and Mona Martyn-Smith BSc for maintenance of the APGI database and biospecimen resource.

\section{Funding/support}

The work was supported by the National Health and Medical Research Council of Australia (NHMRC; 631701, 535903, 535914); Cancer Council NSW (SRP06-01; ICGC09-01; SRP11-01); Australian Government: Department of Innovation, Industry, Science, Research and Tertiary Education (DIISRTE); Australian Cancer Research Foundation (ACRF); Queensland Government (NIRAP); University of Queensland; Cancer Institute NSW (06/ECF/1-24, 09/ CDF/2-40, 07/CDF/1-03, 10/CRF/1-01, 08/RSA/1-15, 10/CDF/2-26,10/FRL/2-03, 06/RSA/1-05, 09/RIG/1-02, 10/TPG/1-04, 11/REG/1-10, 11/CDF/3-26); Garvan Institute of Medical Research; Avner Nahmani Pancreatic Cancer Foundation; R.T. Hall Trust; Jane Hemstritch in memory of Philip Hemstritch; Gastroenterological Society of Australia (GESA); Royal Australasian College of Surgeons (RACS); Sydney Catalyst Translational Cancer Centre; Royal Australasian College of Physicians (RACP); Royal College of Pathologists of Australasia (RCPA) and the St Vincent's Clinic Foundation.

\section{Author details}

'Kinghorn Cancer Centre and Garvan Institute of Medical Research, Darlinghurst, Sydney, Australia. ${ }^{2}$ Anatomical Pathology, Sydpath, St Vincent's Hospital, Sydney, Australia. ${ }^{3}$ St Vincent's Clinical School, University of New South Wales, Sydney, Australia. ${ }^{4}$ Department of Anatomical Pathology, Royal North Shore Hospital, St Lenoards, Sydney, Australia. ${ }^{5}$ Sydney Medical School, University of Sydney, Sydney, Australia. ${ }^{6}$ Queensland Centre for Medical Genomics, Institute for Molecular Bioscience, University of Queensland, Brisbane, Australia. 'Department of Cancer Medicine, Hammersmith Hospital, Imperial College Healthcare NHS Trust, London, UK. ${ }^{8}$ Prince of Wales Clinical School, University of New South Wales and Prince of Wales Hospital, Sydney, Australia. 'Histopath Pathology, 97 Waterloo Road, North Ryde, NSW 2113, Australia. ${ }^{10}$ Macarthur Cancer Therapy Centre, Sydney South West District Health Service, Sydney, NSW, Australia. ${ }^{11}$ Upper Gastrointestinal Surgery Unit, Royal North Shore Hospital, Sydney, Australia. ${ }^{12}$ Wolfson Wohl Cancer Research Centre, Institute of Cancer Sciences, University of Glasgow, Glasgow, UK. ${ }^{13}$ West of Scotland Pancreatic Unit, Glasgow Royal Infirmary, Glasgow, UK.

Received: 11 June 2013 Accepted: 23 August 2013

Published: 31 August 2013

\section{References}

1. Jemal A, Siegel R, Xu J, Ward E: Cancer statistics 2010. CA Cancer J Clin 2010, 60:277-300.

2. Showalter SL, Charles S, Belin J, Cozzitorto J, Einstein P, Richards NG, Sauter PK, Kennedy EP, Witkiewicz A, Brody JR, Yeo CJ: Identifying pancreatic cancer patients for targeted treatment: the challenges and limitations of the current selection process and vision for the future. Expert Opin Drug Deliv 2010, 7:273-284.

3. Biankin AV, Hudson TJ: Somatic variation and cancer: therapies lost in the mix. Hum Genet 2011, 130:79-91.

4. Swanton C, Futreal A, Eisen T: Her2-targeted therapies in non-small cell lung cancer. Clin Cancer Res 2006, 12:4377s-4383s.

5. McAlpine JN, Wiegand KC, Vang R, Ronnett BM, Adamiak A, Kobel M, Kalloger SE, Swenerton KD, Huntsman DG, Gilks CB, Miler DM: HER2 overexpression and amplification is present in a subset of ovarian mucinous carcinomas and can be targeted with trastuzumab therapy. BMC cancer 2009, 9:433.

6. Hudson TJ, Anderson W, Aretz A, et al: International Network of Cancer Genome Projects. Nature 2010, 464:993-998.

7. Hede K: Superhighway or blind alley? The cancer genome atlas releases first results. J Natl Cancer Inst 2008, 100:1566-1569.

8. Samuel N, Hudson TJ: The molecular and cellular heterogeneity of pancreatic ductal adenocarcinoma. Nature Rev Gastroenterol Hepatol 2012, 9:77-87.
9. Biankin AV, Waddell N, Kassahn KS, et al: Pancreatic cancer genomes reveal aberrations in axon guidance pathway genes. Nature 2012, 491:399-405.

10. Jones S, Zhang X, Parsons DW, Lin JC, Leary RJ, Angenendt P, Mankoo P, Carter H, Kamlyama H, Jimeno A, Hong SMFB, Lin MT, Calhoun ES, Kamiyama M, Walter K, Nikolskaya T, Nikolsky Y, Hartigan J, Smith DR, Hidalgo M, Leach SD, Klein AP, Jaffee EM, Goggins M, Maitra A, lacobuzioDonahue C, Eshleman JR, Kern SE, Hruban RH, Karchin R, et al: Core signaling pathways in human pancreatic cancers revealed by global genomic analyses. Science 2008, 321:1801-1806.

11. Bang YJ, Van Cutsem E, Feyereislova A, Chung HC, Shen L, Sawaki A, Lordick F, Ohtsu A, Omuro Y, Satoh T, Aprile G, Kulikov E, Hill J, Lehle M, Rüschoff J, Kang YK, ToGA Trial Investigators: Trastuzumab in combination with chemotherapy versus chemotherapy alone for treatment of HER2-positive advanced gastric or gastro-oesophageal junction cancer (ToGA): a phase 3, open-label, randomised controlled trial. Lancet 2010, 376:687-697.

12. Law LY: Dramatic response to trastuzumab and Paclitaxel in a patient with human epidermal growth factor receptor 2-positive metastatic cholangiocarcinoma. J Clin Oncol 2012, 30:e271-e273.

13. Buchler $P$, Reber HA, Buchler MC, Roth MA, Büchler MW, Friess H, Isacoff WH, Hines OJ: Therapy for pancreatic cancer with a recombinant humanized anti-HER2 antibody (herceptin). J Gastrointest Surg 2001, 5:139-146.

14. Kimura K, Sawada T, Komatsu M, Inoue M, Muguruma K, Nishihara T, Yamashita Y, Yamada N, Ohira M, Hirakawa K: Antitumor effect of trastuzumab for pancreatic cancer with high HER-2 expression and enhancement of effect by combined therapy with gemcitabine. Clin Cancer Res 2006, 12:4925-4932.

15. Safran $H$, lannitti $D$, Ramanathan $R$, Schwartz JD, Steinhoff M, Nauman C, Hesketh P, Rathore R, Wolff R, Tantravahi U, Hughes TM, Maia C, Pasquariello T, Goldstein L, King T, Tsai JY, Kennedy T: Herceptin and gemcitabine for metastatic pancreatic cancers that overexpress HER-2/neu. Cancer Invest 2004, 22:706-712.

16. Harder J, Ihorst $G$, Heinemann $V$, Hofheinz R, Moehler M, Buechler P, Kloeppel G, Röcken C, Bitzer M, Boeck S, Endlicher E, Reinacher-Schick A, Schmoor C, Geissler M: Multicentre phase II trial of trastuzumab and capecitabine in patients with HER2 overexpressing metastatic pancreatic cancer. Br J Cancer 2012, 106:1033-1038.

17. Australian Pancreatic Cancer Genome Initiative. www.pancreaticcancer.net.au.

18. Parker JS, Mullins M, Cheang MC, Leung S, Voduc D, Vickery T, Davies S, Fauron C, He X, Hu Z, Quackenbush JF, Stijleman IJ, Palazzo J, Marron JS, Nobel AB, Mardis E, Nielsen TO, Ellis MJ, Perou CM, Bernard PS: Supervised risk predictor of breast cancer based on intrinsic subtypes. $J$ Clin Oncol 2009, 27:1160-1167.

19. Chang DK, Johns AL, Merrett ND, Gill AJ, Colvin EK, Scarlett CJ, Nguyen NQ, Leong RW, Cosman PH, Kelly MI, Sutherland RL, Henshall SM, Kench JG, Biankin AV: Margin clearance and outcome in resected pancreatic cancer. J Clin Oncol 2009, 27:2855-2862.

20. Humphris JL, Chang DK, Johns AL, Scarlett CJ, Pajic M, Jones MD, Colvin EK, Nagrial A, Chin VT, Chantrill LA, Samra JS, Gill AJ, Kench JG, Merrett ND, Das A, Musgrove EA, Sutherland RL, Biankin AV, NSW Pancreatic Cancer Network: The prognostic and predictive value of serum CA19.9 in pancreatic cancer. Annal Oncol 2012, 23:1713-1722.

21. Hofmann M, Stoss $O$, Shi D, Büttner $R$, van de Vijver M, Kim W, Ochiai A, Rüschoff J, Henkel T: Assessment of a HER2 scoring system for gastric cancer: results from a validation study. Histopathology 2008, 52:797-805.

22. Kennecke $H$, Yerushalmi R, Woods R, Cheang MC, Voduc D, Speers CH, Nielsen TO, Gelmon K: Metastatic behavior of breast cancer subtypes. J Clin Oncol 2010, 28:3271-3277.

23. Smeenk HG, van Eijck CH, Hop WC, Erdmann J, Tran KC, Debois M, van

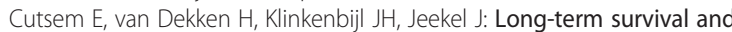
metastatic pattern of pancreatic and periampullary cancer after adjuvant chemoradiation or observation: long-term results of EORTC trial 40891. Ann Surg 2007, 246:734-740.

24. lacobuzio-Donahue CA, Fu B, Yachida S, Luo M, Abe H, Henderson CM, Vilardell F, Wang Z, Keller JW, Banerjee P, Herman JM, Cameron JL, Yeo CJ, Halushka MK, Eshleman JR, Raben M, Klein AP, Hruban RH, Hidalgo M, Laheru D: DPC4 gene status of the primary carcinoma correlates with patterns of failure in patients with pancreatic cancer. J Clin Oncol 2009, 27:1806-1813.

25. Kim ES, Herbst RS, Wistuba II, Lee JJ, Blumenschein GR Jr, Tsao A, Stewart DJ, Hicks ME, Erasmus J Jr, Gupta S, Alden CM, Liu S, Tang X, Khuri FR, Tran 
HT, Johnson BE, Heymach JV, Mao L, Fossella F, Kies MS,

Papadimitrakopoulou V, Davis SE, Lippman SM, Hong WK: The BATTLE trial: personalizing therapy for lung cancer. Cancer Discov 2011, 1:44-53.

26. Esserman $\sqcup$, Berry DA, DeMichele A, Carey L, Davis SE, Buxton M, Hudis C, Gray JW, Perou C, Yau C, Livasy C, Krontiras H, Montgomery L, Tripathy D, Lehman C, Liu MC, Olopade Ol, Rugo HS, Carpenter JT, Dressler L, Chhieng D, Singh B, Mies C, Rabban J, Chen YY, Giri D, Van't Veer L, Hylton N: Pathologic complete response predicts recurrence-free survival more effectively by cancer subset: results from the I-SPY 1 TRIAL-CALGB 150007/150012, ACRIN 6657. J Clin Oncol 2012, 30:3242-3249.

27. Medical Research Council Clinical Trial. http//www.focus4trial.org.

28. Galsky MD, Von Hoff DD, Neubauer M, Anderson T, Fleming M, Nagarwala Y, Mahoney JM, Midwinter D, Vocila L, Zaks TZ: Target-specific, histologyindependent, randomized discontinuation study of lapatinib in patients with HER2-amplified solid tumors. Invest New Drugs 2012, 30:695-701.

doi:10.1186/gm482

Cite this article as: Chou et al.: Clinical and molecular characterization of HER2 amplified-pancreatic cancer. Genome Medicine 2013 5:78.

\section{Submit your next manuscript to BioMed Central and take full advantage of:}

- Convenient online submission

- Thorough peer review

- No space constraints or color figure charges

- Immediate publication on acceptance

- Inclusion in PubMed, CAS, Scopus and Google Scholar

- Research which is freely available for redistribution 